\title{
Longitudinal Study of the Stress Responses of Local Government Workers Who Have Been Impacted by a Natural Disaster
}

\author{
Koubun Wakashima, Ph.D ${ }^{1)}$, Taku Kobayashi, M.A ${ }^{1)}$, Takahiro Kozuka, Ph.D ${ }^{2)}$, \\ Shuji Noguchi M.A ${ }^{1)}$, Michiko Ikuta, Ph.D ${ }^{3)}$, Hideo Ambo, M.A ${ }^{1)}$, \& Keizo Hasegawa, Ph.D ${ }^{1)}$ \\ 1) Graduate School of Education, Tohoku University \\ ${ }^{2)}$ Faculty of Human and Culture Science, Sakushin Gakuin University \\ ${ }^{3)}$ Kanagawa University of Human Services
}

ABSTRACT. We conducted 4 times surveys starting from June 2011 and continuing through February 2013, in order to check up stress response of local government workers who suffered The Great East Japan Earthquake (Higashi Nihon Daishinsai) with K6 and Comprehensive Stress Response Inventory (CSI). Our study has three aims. First, we will organize the chronological pattern of stress responses. Second, we will examine how the initial disastrous condition and attributes of survey participants relate to the chronological pattern of subsequent stress responses. Third, we will examine how the current stress responses interplay with the chronological pattern of stress responses.

Result of this study show that (1) chronological patterns of stress response are classified into 8 clusters (no-stress response maintaining group, low-stress response maintaining group, low-stress response to no-stress response slow improvement group, middle-stress response to high-stress response slow deteriorated group, middle-stress response to low-stress response slow improvement group, low-stress response to no-stress response rapid improvement group, high-stress response to low-stress response rapid improvement group, severe-stress response maintaining group — $-13.55 \%$ of local government workers remained at a high level of stress 15 months after the earthquake), (2) damage of domicile and living outside their domiciles relate to stress response of local government workers, (3) two factors of CSI (stress response to a disaster and autonomic symptoms) relate with state at the outset of disaster and other two factors of CSI (anxiety / tense and displeasure / anger) relate with recent conditions. We mainly discussed two themes about point of attention in mental health care for local government workers at a natural disaster and points of attention in assessment of PTSD-like response.

KEY WORDS: Great East Japan Earthquake (Higashi Nihon Daishinsai), PTSD, local government workers, Comprehensive Stress Response Inventory

\section{Introduction}

This study is primarily designed to examine

CORRESPONDENCE TO: WAKASHIMA Graduate School

of Education, Tohoku University, 27-1 Kawauchi, Aoba-ku,

Sendai-city, 980-8576, JAPAN.

e-mail: rxv11606@hotmail.co.jp the employees in the regions where the Great East Japan Earthquake (Higashi Nihon Daishinsai) and subsequent tsunamis caused a great impact of damage and destruction. Longitudinal surveys have been conducted 4 times over a total of two years and as a part of the health checkups which are regularly offered 
by the local government sectors to their employees. Because the format of the health checkup needed to be kept simple and concise, we took great consideration of the mental and physical stress that the participant may experience during our survey, and the survey was designed to minimize such stress.

The types of stressful conditions that are experienced by public servants in the locations impacted by the disaster are grouped into three categories as described below (Wakashima \& Noguchi, 2013): (1) Public servants themselves were victims of the disaster: In the early time period following the earthquake and tsunami, some local government workers could not find their own family members, and yet they dedicated their time and effort to supporting their community and its residents. Some workers had lost their homes and family, and yet they served others who had been impacted by the disaster. However, it is considered that even though those service workers may have been mentally hurt, they preferred to continue their activities to serve others instead of becoming inactive and disheartened as victims of the disaster. (2) The increased workload due to the increased needs of victims and public expectations concerning the restoration of towns and cities: From the perspective of the long-term influence on the public servants, the increased workload is one of the most difficult and important issues. When the mental health of the local government workers is considered, the question of how quickly those workers will be able to achieve the restoration of the town while they also maintain the same amount of workload as before the incident should be discussed. (3) The increase amount of complaints/concerns received from residents: Due to the consciousness of their professional responsibilities as public servants, there is the potential risk that local government workers may lose themselves in the significance in their jobs because of the constant complaints and demands that are continuously received from residents, and this may cause them to lose confidence in themselves.

Under the stressful conditions described above, local government workers have been playing a central role in the recovery and restoration of their towns. In this regard, the purpose of our study is to focus on those workers as our subjects and to examine their responses to stressors in following the occurrence of a natural disaster in chronological order. Our study is also aimed at clarifying several aspects.

First, we will organize the chronological pattern of stress responses. Second, we will examine how the initial disastrous condition and attributes of survey participants relate to the chronological pattern of subsequent stress responses. Third, we will examine how the current stress responses interplay with the chronological pattern of stress responses.

\section{Methods}

1. Research participants and investigation time

Our research participants are municipal government workers who serve at local city halls on the coast of the Tohoku region (the northeastern area of Honshu). The number of 
the participants in each survey varies from 1,378 to 1,452 persons. We conducted our surveys four times starting from June 2011 (which was the year that the Great East Japan Earthquake occurred) and continuing through February 2013. Table 1, shown below, shows the number of participants for each survey, their gender and ages, and the dates that we conducted each survey.

Specific questions - whether or not the subjects were involved with disaster-related duties, the extent of damage to their domiciles, whether or not they experienced life without a domicile, and whether or not their family members were missing or dead-were considered to be constant and asked only once at the $1^{\text {st }}$ investigation.

\section{The content of our survey}

The survey is composed of basic demographic questions including age, gender, job location and title, and whether or not participants would request to see mental health clinicians such as psychiatrists or clinical psychotherapists. In addition, the survey also includes questions about the condition of participant's workplace, the damage to their personal property, and their physical and mental health conditions.

1) Condition of Workplace (This portion of the survey was conducted only once at the $1^{\text {st }}$ investigation.)

(1) Whether or not subjects were involved with disaster-related duties

1. Handling victims' bodies and related operations

2. Handling complaints from residents

3. Others

4. No

5. More than one duty

(2) Whether or not subjects have worked overtime exceeding 100 hours.

(1. Yes 2. No)

(3) Communication with co-workers at their workplace

(1. Yes, regularly 2. Yes, occasionally 3. No, not at all)

2) Situation of personal damage caused by

\begin{tabular}{|c|c|c|}
\hline & Date & Number of participants \\
\hline \multirow{2}{*}{ 1st investigation } & June 2011 & $\begin{array}{c}1452 \text { (male:806, female:646) } \\
\text { Mean of age :45.09 }\end{array}$ \\
\hline \multirow{2}{*}{ 2nd investigation } & October 2011 & $\begin{array}{c}1414 \text { (male804, female:610) } \\
\text { Mean of age :45.46 }\end{array}$ \\
\hline \multirow{2}{*}{ 3rd investigation } & June 2012 & $\begin{array}{c}1387 \text { (male:797, female:590) } \\
\text { Mean of age:44.02 }\end{array}$ \\
\hline \multirow{2}{*}{ 4th investigation } & February 2013 & $\begin{array}{c}1378 \text { (male: } 799, \text { female:579) } \\
\text { Mean of age:44.81 }\end{array}$ \\
\hline
\end{tabular}


the disaster (This portion of the survey was conducted only once at the $1^{\text {st }}$ investigation.)

(1) Damage to domicile

1. Completely collapsed

2. Half-collapsed/largely destroyed

3. Partially destroyed

4. Slightly damaged

5. No damage

(2) Whether or not subjects had the experience of living without a domicile

(e.g. staying at an evacuation shelter)

1. Yes I have but not now

2. Yes, I still live in an arranged situation

3. No, not at all

(3) Whether or not family members have been killed or missing.

(1. Yes 2. No)

3) Health condition (It was conducted from the first through the fourth investigations.)

(1) Physical condition

(1. Good 2.Neither good nor bad 3. Not so well 4. Bad)

(2) Sleep

(1. Very well 2. Well mostly 3. Poorly 4 . Very poorly)

(3) Appetite

(1. Increased 2. Same as last time/before 3. Decreased)

(4) Change in the amount of alcohol beverage consumption

1. Increased

2. Same as last time/before

3. Decreased

4. Not consumption at all)

(5) Whether or not subjects have a chronic disease and status of treatment

1. I managed to have continual treatment

2. Treatment was suspended in the past, but it has resumed

3. Treatment is still been suspended

4) Assessments of mental health condition

(1) K6 in the Japanese version (performed in the $1^{\text {st }}$ through $4^{\text {th }}$ investigations): We used the Japanese version of K6 that was originally created by Kessler, Andrews, Colpe et al. (2002) and designed for the purpose of screening for mental disorders and examining the severity of the disorder (Furukawa, Ono, Uda, \& Nakane, 2003). The Japanese K6 is composed of six items and asks the subject to answer using five point scales ( 0 : not at all through 4: always).

(2) CSI (Comprehensive Stress Response Inventory) (performed at the $4^{\text {th }}$ investigation): CSI was developed by Asai, Morikawa, Hiraizumi, Usami, and Wakashima (2013) in order to easily detect and simultaneously differentiate a type of disaster-specific stress and a type of general stress. It is a comprehensible assessment to measure overall stress responses. The CSI identifies four factors of stress response and is composed of 25 items in total. Three factors are each categorized into "anxiety/tense," "displeasure/anger," and "autonomic symptoms" and considered to describe as the general-stress response. The other factor is considered as a "specific stress response to a disaster". Symptoms for the factor include flashbacks of intrusive memories, avoidance of activities that stimulate the 
traumatic event, and re-experiencing the trauma.

Furthermore, the CSI examines one's general-stress response by excluding the specific stress response to a disaster and measuring three other factors. As for examination of PTSD and alike, it includes all four stress factors (the sum of the four factors). Particularly in this study, we presupposed the Great East Japan Earthquake as the specific disaster for the assessment of the specific stress response and asked each item in four point scales starting from the lowest scale, "1: Never," to the highest scale, "4: Most of the time." Likewise, in terms of the general stress response, we asked participants about their observed symptoms during the last 30 days, and each question was scaled from "1: Never" to "4" Most of the time."

\section{Scoring of variables}

This section describes the scoring of variables. However, it excludes the items that we did not include in our investigation of study subjects, such as physical condition, appetite, the amount of alcohol beverage consumption, and the presence of chronic disease and condition for its treatment. In regard to the following items - experience of disaster-related duties, overtime exceeding 100 hours of work, the degree of domicile damage, arrangements for living outside the home, whether or not family members are missing or dead, and gender, we used the response categories of each question as categorical variables. On the other hand, regarding questions involving communication with co-workers (1 item), sleep (1 item), K6 in the Japanese version (6 items), CSI (8 items of specific stress response to a disaster, 9 items of anxiety/tense factors, 4 items of displeasure/anger factors, 4 items of autonomic symptom factors, for a total of 25 items), rating scales values and their total values were chosen for scoring. These quantitative data are set such that a higher score indicate a worse condition of the participant.

\section{Results}

For the purpose of organizing the stress response patterns in a chronological order, we placed the values of "communication with co-workers," "asleep condition," and "scores of K6" that were collected from the 1 st investigation through the 3 rd investigation into categorical variables and conducted a cluster analysis in accordance with the Ward method. The number of subjects from the $1^{\text {st }}$ investigation through $3^{\text {rd }}$ investigation was 952 persons and there were no missing values during the series of investigations. Because we valued perspective interpretability, a structure of 8 clusters was established for this analysis.

Each cluster for analysis is termed as follows: 1) No-stress response maintaining group (n=291), 2) Low-stress response maintaining group $(\mathrm{n}=168)$, 3) Low-stress response to no- stress response slow improvement group ( $\mathrm{n}=130)$, 4) Middle-stress response to high-stress response slow deteriorated group ( $\mathrm{n}=87)$, 5) Middle-stress response to low-stress response slow improvement group $(\mathrm{n}=76)$, 6) Low-stress 
response to no-stress response rapid cluster are described in Table 2, and descriptive improvement group ( $\mathrm{n}=92)$, 7) High-stress statistics are shown from Tables 3 through response to low stress response rapid Table 5, followed by the profile of each cluster improvement group $(\mathrm{n}=66)$, and 8) in Figure 1.

Severe-stress response maintaining group

In regard to naming clusters, we referred $(n=42)$. In order to explain the foundation of classification of Wakashima, Kozuka, Noguchi naming each cluster, particular features of each et al. (2012). In this study, we took score of K6

Table2. Feature of Each Cluster

\begin{tabular}{|c|c|c|}
\hline Cluster & $\begin{array}{l}\text { Number } \\
\text { of people }\end{array}$ & Feature \\
\hline $\begin{array}{l}\text { No-stress response } \\
\text { maintaining group }\end{array}$ & 291 & $\begin{array}{l}\text { On the whole, this cluster is better than other clusters } \\
\text { from 1st investigation through } 3 \text { rd investigation. }\end{array}$ \\
\hline $\begin{array}{l}\text { Low-stress response } \\
\text { maintaining group }\end{array}$ & 168 & $\begin{array}{l}\text { Stress response had deteriorated from } 2 \text { nd investigation } \\
\text { to } 3 \text { rd investigation. But, on the whole, this cluster not } \\
\text { has much of a problem. }\end{array}$ \\
\hline $\begin{array}{l}\text { Low-stress response to } \\
\text { no- stress response slow } \\
\text { improvement group }\end{array}$ & 130 & $\begin{array}{l}\text { This cluster's problems slight-to-moderate at the outset } \\
\text { of disaster, but these problems improved from } 2 \mathrm{nd} \\
\text { investigation to 3rd investigation. }\end{array}$ \\
\hline $\begin{array}{l}\text { Middle-stress response to } \\
\text { high-stress response slow } \\
\text { deteriorated group }\end{array}$ & 87 & $\begin{array}{l}\text { Asleep condition is bad from } 1 \text { st investigation through } \\
3 \text { rd investigation, and stress response had deteriorated } \\
\text { from } 2 \text { nd investigation to } 3 \text { rd investigation in this } \\
\text { cluster. }\end{array}$ \\
\hline $\begin{array}{l}\text { Middle-stress response to } \\
\text { low-stress response slow } \\
\text { improvement group }\end{array}$ & 76 & $\begin{array}{l}\text { This cluster had moderate problems at the outset of } \\
\text { disaster, but problems improved from } 2 \text { nd investigation } \\
\text { to } 3 \text { rd investigation. }\end{array}$ \\
\hline $\begin{array}{l}\text { Low-stress response to } \\
\text { no-stress response rapid } \\
\text { improvement group }\end{array}$ & 92 & $\begin{array}{l}\text { This cluster had slight-to-moderate problems at the } \\
\text { outset of disaster, but these problems had improved at } \\
\text { 2nd investigation. }\end{array}$ \\
\hline $\begin{array}{l}\text { High-stress response to } \\
\text { low stress response rapid } \\
\text { improvement group }\end{array}$ & 66 & $\begin{array}{l}\text { This cluster had severe problems at the outset of } \\
\text { disaster. Improvement in the problem of stress response } \\
\text { is remarkable. Problems of asleep condition and } \\
\text { communication improved too at 2nd investigation. }\end{array}$ \\
\hline $\begin{array}{l}\text { Severe-stress response } \\
\text { maintaining group }\end{array}$ & 42 & $\begin{array}{l}\text { This cluster is worst in all clusters with respect to all } \\
\text { indicators of condition of health. }\end{array}$ \\
\hline Total & 952 & \\
\hline
\end{tabular}


Table3. Descriptive Statistics about Asleep Condition

\begin{tabular}{|c|c|c|c|c|c|c|}
\hline & \multicolumn{2}{|c|}{ Asleep condition (1) } & \multicolumn{2}{|c|}{ Asleep condition(2) } & \multicolumn{2}{|c|}{ Asleep condition (3) } \\
\hline & $M$ & $S D$ & $M$ & $S D$ & $M$ & $S D$ \\
\hline $\begin{array}{l}\text { No-stress response maintaining } \\
\text { group }\end{array}$ & 1.80 & .55 & 1.67 & .53 & 1.73 & .55 \\
\hline $\begin{array}{l}\text { Low-stress response maintaining } \\
\text { group }\end{array}$ & 2.14 & .56 & 2.02 & .60 & 2.15 & .53 \\
\hline $\begin{array}{l}\text { Low-stress response to no- stress } \\
\text { response slow improvement group }\end{array}$ & 2.02 & .53 & 2.09 & .49 & 1.86 & .53 \\
\hline $\begin{array}{l}\text { Middle-stress response to high- } \\
\text { stress response slow deteriorated }\end{array}$ & 2.34 & .59 & 2.25 & .61 & 2.46 & .66 \\
\hline $\begin{array}{l}\text { Middle-stress response to low-stress } \\
\text { response slow improvement group }\end{array}$ & 2.47 & .62 & 2.50 & .62 & 2.24 & .46 \\
\hline $\begin{array}{l}\text { Low-stress response to no-stress } \\
\text { response rapid improvement group }\end{array}$ & 2.13 & .63 & 1.83 & .51 & 1.82 & .47 \\
\hline $\begin{array}{l}\text { High-stress response to low stress } \\
\text { response rapid improvement group }\end{array}$ & 2.32 & .66 & 1.95 & .59 & 2.00 & .46 \\
\hline $\begin{array}{l}\text { Severe-stress response maintaining } \\
\text { group }\end{array}$ & 2.76 & .79 & 2.74 & .77 & 2.60 & .77 \\
\hline Total & 2.10 & .64 & 1.99 & .64 & 1.99 & .61 \\
\hline
\end{tabular}

Note: Each numbers framed by circle indicate the time of investigation.

Table4. Descriptive Statistics about K6

\begin{tabular}{|c|c|c|c|c|c|c|}
\hline & \multicolumn{2}{|c|}{ K6(1) } & \multicolumn{2}{|c|}{ K6(2) } & \multicolumn{2}{|c|}{ K6(3) } \\
\hline & $M$ & $S D$ & $M$ & $S D$ & $M$ & $S D$ \\
\hline $\begin{array}{l}\text { No-stress response maintaining } \\
\text { group }\end{array}$ & 2.43 & 1.76 & 1.31 & 1.35 & 1.58 & 1.68 \\
\hline $\begin{array}{l}\text { Low-stress response maintaining } \\
\text { group }\end{array}$ & 6.24 & 2.31 & 4.67 & 2.50 & 7.41 & 2.18 \\
\hline $\begin{array}{l}\text { Low-stress response to no- stress } \\
\text { response slow improvement group }\end{array}$ & 5.42 & 2.34 & 6.48 & 1.37 & 2.97 & 1.63 \\
\hline $\begin{array}{l}\text { Middle-stress response to high- } \\
\text { stress response slow deteriorated }\end{array}$ & 10.16 & 2.82 & 9.45 & 2.19 & 12.69 & 4.14 \\
\hline $\begin{array}{l}\text { Middle-stress response to low-stress } \\
\text { response slow improvement group }\end{array}$ & 9.55 & 2.83 & 12.45 & 3.07 & 6.55 & 3.07 \\
\hline $\begin{array}{l}\text { Low-stress response to no-stress } \\
\text { response rapid improvement group }\end{array}$ & 8.80 & 2.10 & 2.86 & 1.57 & 1.78 & 1.73 \\
\hline $\begin{array}{l}\text { High-stress response to low stress } \\
\text { response rapid improvement group }\end{array}$ & 14.03 & 3.46 & 6.61 & 2.15 & 5.27 & 3.21 \\
\hline $\begin{array}{l}\text { Severe-stress response maintaining } \\
\text { group }\end{array}$ & 17.40 & 3.89 & 16.43 & 3.68 & 14.26 & 4.60 \\
\hline Total & 6.86 & 4.76 & 5.43 & 4.60 & 5.05 & 4.67 \\
\hline
\end{tabular}

Note: Each numbers framed by circle indicate the time of investigation. 
as the criterion of severity of stress response. A range of 0 to 3 is defined as "no-stress response", 4 to 9 is defined as "low-stress response", 10 to 12 is defined as "middle-stress response", and not lower than 13 is defined as "high-stress response" or "severe-stress response". Initial expression of each cluster mentions degree of stress response at $1^{\text {st }}$ investigation. Depending on changes over time, we added to corresponding words (maintaining, improvement, or deteriorated). Furthermore, "rapid" and "slow" indicate the moment when severity of stress response changed. "Rapid" means that changes resulted between $1^{\text {st }}$ investigation and $2^{\text {nd }}$ investigation. "Slow" means that changes resulted between $2^{\text {nd }}$ investigation and $3^{\text {rd }}$ investigation.

Next, we conducted a chi-square test for those clusters regarding conditions of the disaster and chronological patterns. The purpose of this test is to examine how the initial disaster condition and attributes of the subjects (i.e. involvement of disaster-related duties, more than 100 hours of overtime work, experiences of domicile damage and outside domicile life, dead or missing person(s) in the family, and gender) are related with their subsequent stress responses in chronological patterns.

As a result, no statistical significance was found in the aspects of involvement of disaster-related duties $\left(\chi^{2}=38.01, d f=28, n . s.\right)$, more than 100 hours of overtime work $\left(\chi^{2}=14.06, d f=7, n . s.\right)$, and gender $\left(\chi^{2}=8.25, d f\right.$ $=7, n . s$.$) . On the other hand, significant$ differences were found in the variables of damage to a domicile $\left(\chi^{2}=51.39, d f=28, p\right.$ $<.01)$, experiences of life outside a domicile $(\chi$ $\left.{ }^{2}=40.44, d f=14, p<.01\right)$, and having dead or missing person(s) in the family $\left(\chi^{2}=18.58, d f=\right.$ $7, p<.01)$. In accordance with these results, we also conducted residual analyses for the

Table5. Descriptive Statistics about Communication with Co-worker

\begin{tabular}{|c|c|c|c|c|c|c|}
\hline & \multicolumn{2}{|c|}{ COM(1) } & \multicolumn{2}{|c|}{ COM(2) } & \multicolumn{2}{|c|}{ COM(3) } \\
\hline & $M$ & $S D$ & $M$ & $S D$ & $M$ & $S D$ \\
\hline $\begin{array}{l}\text { No-stress response maintaining } \\
\text { group }\end{array}$ & 1.78 & .49 & 1.79 & .46 & 1.54 & .51 \\
\hline $\begin{array}{l}\text { Low-stress response maintaining } \\
\text { group }\end{array}$ & 1.90 & .46 & 1.88 & .37 & 1.80 & .50 \\
\hline $\begin{array}{l}\text { Low-stress response to no- stress } \\
\text { response slow improvement group }\end{array}$ & 1.88 & .45 & 1.92 & .41 & 1.58 & .49 \\
\hline $\begin{array}{l}\text { Middle-stress response to high- } \\
\text { stress response slow deteriorated }\end{array}$ & 1.97 & .59 & 2.03 & .61 & 1.91 & .45 \\
\hline $\begin{array}{l}\text { Middle-stress response to low-stress } \\
\text { response slow improvement group }\end{array}$ & 1.87 & .53 & 1.96 & .47 & 1.61 & .52 \\
\hline $\begin{array}{l}\text { Low-stress response to no-stress } \\
\text { response rapid improvement group }\end{array}$ & 1.98 & .44 & 1.85 & .42 & 1.50 & .52 \\
\hline $\begin{array}{l}\text { High-stress response to low stress } \\
\text { response rapid improvement group }\end{array}$ & 1.94 & .46 & 1.85 & .40 & 1.70 & .55 \\
\hline $\begin{array}{l}\text { Severe-stress response maintaining } \\
\text { group }\end{array}$ & 1.98 & .64 & 2.07 & .56 & 1.98 & .64 \\
\hline Total & 1.88 & .49 & 1.88 & .44 & 1.66 & .53 \\
\hline
\end{tabular}

Note: Each numbers framed by circle indicate the time of investigation."COM" indicate communication with co־worker. 


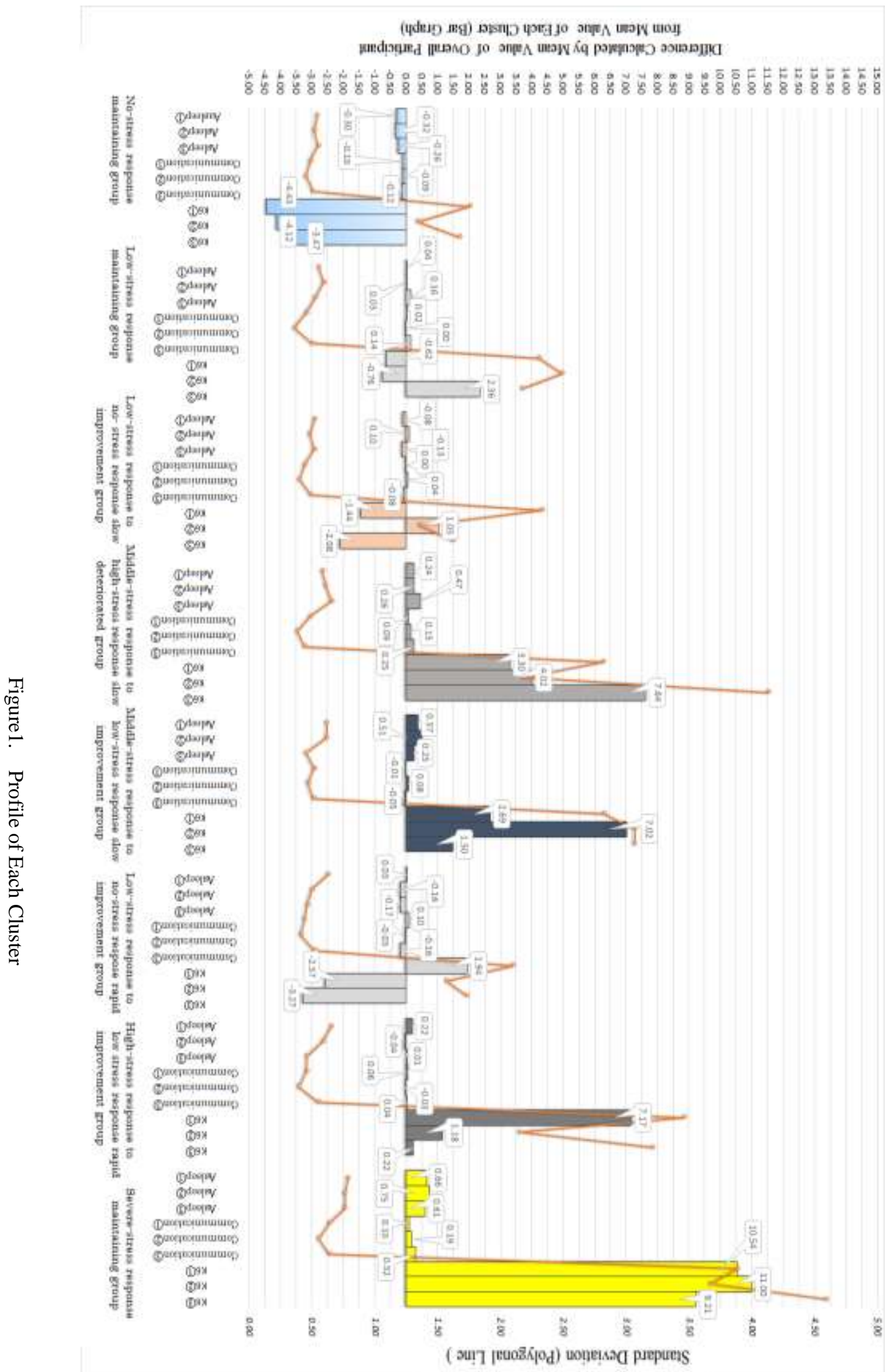


variables that statistical significance was observed. The results of residual analyses, which are itemized in damage of a domicile, experience of life outside the domicile, and having dead or missing person(s) in the family are indicated in Tables 6 through 11.
According to the result of residual analysis based on a cross-tabulation table between the cluster of the damage of domiciles and the cluster of chronological patterns, the subjects whose domiciles were completely destroyed have the highest probability to be placed in the

Table6. Result of Residual Analysis about Damage of Domicile $(n=944)$

\begin{tabular}{|c|c|c|c|c|c|c|c|}
\hline Cluster & & Complete collapse & Half collapse & Partially destroy & Slightly damage & No damage & $x^{2}$ \\
\hline \multirow{3}{*}{$\begin{array}{l}\text { No-stress response } \\
\text { maintaining group }\end{array}$} & Frequency & 50 & 39 & 98 & 74 & 27 & \\
\hline & $\begin{array}{l}\text { Expected } \\
\text { frequency }\end{array}$ & 68.95 & 44.85 & 89.08 & 63.76 & 21.36 & \\
\hline & Adj.residual & -3.14 & -1.14 & 1.36 & 1.74 & 1.52 & \\
\hline \multirow{3}{*}{$\begin{array}{l}\text { Low-stress response } \\
\text { maintaining group }\end{array}$} & Frequency & 35 & 25 & 54 & 40 & 13 & \\
\hline & $\begin{array}{l}\text { Expected } \\
\text { frequency }\end{array}$ & 39.98 & 26.01 & 51.66 & 36.97 & 12.38 & \\
\hline & Adj.residual & -1.00 & .24 & .43 & .62 & .20 & \\
\hline \multirow{3}{*}{$\begin{array}{l}\text { Low-stress response to } \\
\text { no- stress response } \\
\text { slow improvement } \\
\text { group }\end{array}$} & Frequency & 33 & 28 & 36 & 22 & $\overline{9}$ & \\
\hline & $\begin{array}{l}\text { Expected } \\
\text { frequency }\end{array}$ & 30.64 & 19.93 & 39.59 & 28.34 & 9.49 & \\
\hline & Adj.residual & .52 & 2.12 & $: 74$ & -1.45 & $: 18$ & \\
\hline \multirow{3}{*}{$\begin{array}{l}\text { Middle-stress response } \\
\text { to high-stress } \\
\text { response slow } \\
\text { deteriorated group }\end{array}$} & Frequency & 29 & 13 & 22 & 19 & 4 & \\
\hline & $\begin{array}{l}\text { Expected } \\
\text { frequency }\end{array}$ & 20.83 & 13.55 & 26.91 & 19.26 & 6.45 & \\
\hline & Adj.residual & 2.15 & -0.17 & -1.20 & -0.07 & -1.05 & \\
\hline \multirow{3}{*}{$\begin{array}{l}\text { Middle-stress response } \\
\text { to low-stress response } \\
\text { slow improvement } \\
\text { group }\end{array}$} & Frequency & 18 & 8 & 27 & 18 & 4 & \\
\hline & $\begin{array}{l}\text { Expected } \\
\text { frequency }\end{array}$ & 17.96 & 11.68 & 23.20 & 16.60 & 5.56 & $51.39(28)^{* *}$ \\
\hline & Adj.residual & .01 & -1.22 & .99 & .40 & $\cdot .72$ & \\
\hline \multirow{3}{*}{$\begin{array}{l}\text { Low-stress response to } \\
\text { no-stress response } \\
\text { rapid improvement } \\
\text { group }\end{array}$} & Frequency & 18 & 16 & 31 & 19 & 7 & \\
\hline & $\begin{array}{l}\text { Expected } \\
\text { frequency }\end{array}$ & 21.79 & 14.17 & 28.15 & 20.15 & 6.75 & \\
\hline & Adj.residual & .98 & .56 & .68 & $: 30$ & .11 & \\
\hline \multirow{3}{*}{$\begin{array}{l}\text { High-stress response } \\
\text { to low stress response } \\
\text { rapid improvement } \\
\text { group }\end{array}$} & Frequency & 26 & 6 & 17 & 11 & $\overline{6}$ & \\
\hline & $\begin{array}{l}\text { Expected } \\
\text { frequency }\end{array}$ & 15.80 & 10.28 & 20.42 & 14.61 & 4.89 & \\
\hline & Adj.residual & 3.05 & -1.51 & .94 & -1.11 & .54 & \\
\hline \multirow{3}{*}{$\begin{array}{l}\text { Severe-stress respons } \\
\text { maintaining group }\end{array}$} & Frequency & 17 & 12 & 7 & 6 & $\overline{0}$ & \\
\hline & $\begin{array}{l}\text { Expected } \\
\text { frequency }\end{array}$ & 10.06 & 6.54 & 12.99 & 9.30 & 3.11 & \\
\hline & Adj.residual & 2.57 & 2.38 & -2.05 & -1.25 & -1.88 & \\
\hline \multirow[b]{2}{*}{ Total } & Frequency & 226 & 147 & 292 & 209 & 70 & \\
\hline & $\begin{array}{l}\text { Expected } \\
\text { frequency }\end{array}$ & 226.0 & 147.0 & 292.0 & 209.0 & 70.0 & \\
\hline
\end{tabular}

${ }_{* *}^{*} p<.01$ 
Table7. Abstract about Residual Analysis on

Damage to Domicile $(n=944)$

\begin{tabular}{|c|c|c|}
\hline \multirow[t]{2}{*}{ Complete collapse } & $\begin{array}{c}\text { Great } \\
\text { frequency }\end{array}$ & $\begin{array}{l}\text { Middle-stress response to high-stress response slow } \\
\text { deteriorated group * } \\
\text { High-stress response to low stress response rapid } \\
\text { improvement group } * * \\
\text { Severe-stress response maintaining group } *\end{array}$ \\
\hline & $\begin{array}{c}\text { Low } \\
\text { frequency }\end{array}$ & No-stress response maintaining group $* *$ \\
\hline \multirow[t]{2}{*}{ Half collapse } & $\begin{array}{l}\text { Great } \\
\text { frequency }\end{array}$ & $\begin{array}{l}\text { Low-stress response to no- stress response slow } \\
\text { improvement group * } \\
\text { Severe-stress response maintaining group * }\end{array}$ \\
\hline & $\begin{array}{l}\text { Low } \\
\text { frequency }\end{array}$ & Not available \\
\hline \multirow{2}{*}{ Partially destroy } & $\begin{array}{c}\text { Great } \\
\text { frequency }\end{array}$ & Not available \\
\hline & $\begin{array}{c}\text { Low } \\
\text { frequency }\end{array}$ & Severe-stress response maintaining group $*$ \\
\hline \multirow{2}{*}{ Slightly damage } & $\begin{array}{c}\text { Great } \\
\text { frequency }\end{array}$ & Not available \\
\hline & $\begin{array}{l}\text { Low } \\
\text { frequency }\end{array}$ & Not available \\
\hline \multirow{2}{*}{ No damage } & $\begin{array}{c}\text { Great } \\
\text { frequency }\end{array}$ & Not available \\
\hline & $\begin{array}{c}\text { Low } \\
\text { frequency }\end{array}$ & Not available \\
\hline
\end{tabular}

$* p<.05, * * p<.01$

Table8. Result of Residual Analysis about Experience of Outside Domicile Life $(n=919)$

\begin{tabular}{|c|c|c|c|c|c|}
\hline Cluster & & In past days & $\begin{array}{c}\text { Until 1st } \\
\text { investigation }\end{array}$ & Never & $x^{2}$ \\
\hline \multirow{3}{*}{$\begin{array}{l}\text { No-stress response } \\
\text { maintaining group }\end{array}$} & Frequency & 95 & 29 & 154 & \multirow{26}{*}{$40.44(14)^{* *}$} \\
\hline & $\begin{array}{l}\text { Expected } \\
\text { frequency }\end{array}$ & 110.41 & 36.91 & 130.68 & \\
\hline & Adj.residual & -2.26 & -1.67 & 3.36 & \\
\hline \multirow{3}{*}{$\begin{array}{l}\text { Low-stress response } \\
\text { maintaining group }\end{array}$} & Frequency & 55 & 20 & 87 & \\
\hline & $\begin{array}{l}\text { Expected } \\
\text { frequency }\end{array}$ & 64.34 & 21.51 & 76.15 & \\
\hline & Adj.residual & -1.65 & -0.38 & 1.88 & \\
\hline \multirow{3}{*}{$\begin{array}{l}\text { Low-stress response to } \\
\text { no- stress response } \\
\text { slow improvement } \\
\text { group }\end{array}$} & Frequency & 54 & 14 & 57 & \\
\hline & $\begin{array}{l}\text { Expected } \\
\text { frequency }\end{array}$ & 49.65 & 16.59 & 58.76 & \\
\hline & Adj.residual & 0.86 & -0.74 & -0.34 & \\
\hline \multirow{3}{*}{$\begin{array}{l}\text { Middle-stress response } \\
\text { to high-stress } \\
\text { response slow } \\
\text { deteriorated group }\end{array}$} & Frequency & 35 & 20 & $\overline{31}$ & \\
\hline & $\begin{array}{l}\text { Expected } \\
\text { frequency }\end{array}$ & 34.16 & 11.42 & 40.43 & \\
\hline & Adj.residual & 0.20 & 2.87 & -2.14 & \\
\hline \multirow{3}{*}{$\begin{array}{l}\text { Middle-stress response } \\
\text { to low-stress response } \\
\text { slow improvement } \\
\text { group }\end{array}$} & Frequency & 35 & 7 & 32 & \\
\hline & $\begin{array}{l}\text { Expected } \\
\text { frequency }\end{array}$ & 29.39 & 9.82 & 34.79 & \\
\hline & Adj.residual & 1.39 & -1.01 & -0.68 & \\
\hline \multirow{3}{*}{$\begin{array}{l}\text { Low-stress response to } \\
\text { no-stress response } \\
\text { rapid improvement } \\
\text { group }\end{array}$} & Frequency & 45 & 8 & 36 & \\
\hline & $\begin{array}{l}\text { Expected } \\
\text { frequency }\end{array}$ & 35.35 & 11.82 & 41.84 & \\
\hline & Adj.residual & 2.20 & -1.25 & -1.30 & \\
\hline \multirow{3}{*}{$\begin{array}{l}\text { High-stress response } \\
\text { to low stress response } \\
\text { rapid improvement } \\
\text { group }\end{array}$} & Frequency & 30 & 13 & 20 & \\
\hline & $\begin{array}{l}\text { Expected } \\
\text { frequency }\end{array}$ & 25.02 & 8.36 & 29.61 & \\
\hline & Adj.residual & 1.33 & 1.78 & -2.51 & \\
\hline \multirow{3}{*}{$\begin{array}{l}\text { Severe-stress response } \\
\text { maintaining group }\end{array}$} & Frequency & 16 & 11 & $\overline{15}$ & \\
\hline & $\begin{array}{l}\text { Expected } \\
\text { frequency }\end{array}$ & 16.68 & 5.58 & 19.74 & \\
\hline & Adj.residual & -0.22 & 2.53 & -1.50 & \\
\hline \multirow[b]{2}{*}{ Total } & Frequency & 365 & 122 & 432 & \\
\hline & $\begin{array}{l}\text { Expected } \\
\text { frequency }\end{array}$ & 365.0 & 122.0 & 432.0 & \\
\hline
\end{tabular}


Table9. Abstract about Residual Analysis on

Experience of Outside Domicile Life $(n=919)$

\begin{tabular}{|c|c|c|}
\hline \multirow{2}{*}{ In past days } & $\begin{array}{l}\text { Great } \\
\text { frequency }\end{array}$ & $\begin{array}{l}\text { Low-stress response to no-stress response rapid } \\
\text { improvement group* }\end{array}$ \\
\hline & $\begin{array}{l}\text { Low } \\
\text { frequency }\end{array}$ & No-stress response maintaining group $*$ \\
\hline \multirow[t]{2}{*}{ Until 1st investigation } & $\begin{array}{c}\text { Great } \\
\text { frequency }\end{array}$ & $\begin{array}{l}\text { Middle-stress response to high-stress response slow } \\
\text { deteriorated group } * * \\
\text { Severe-stress response maintaining group } *\end{array}$ \\
\hline & $\begin{array}{c}\text { Low } \\
\text { frequency }\end{array}$ & Not available \\
\hline \multirow[b]{2}{*}{ Never } & $\begin{array}{l}\text { Great } \\
\text { frequency }\end{array}$ & No-stress response maintaining group $* *$ \\
\hline & $\begin{array}{l}\text { Low } \\
\text { frequency }\end{array}$ & $\begin{array}{l}\text { Middle-stress response to high-stress response slow } \\
\text { deteriorated group* } \\
\text { High-stress response to low stress response rapid } \\
\text { improvement group* }\end{array}$ \\
\hline
\end{tabular}

$* p<.05, * * p<.01$

Table10. Result of Residual Analysis about Existence or Non-existence of The Dead / Missing Person in Familly $(\mathrm{n}=937)$

\begin{tabular}{|c|c|c|c|c|}
\hline Cluster & & There is & There is not & $x^{2}$ \\
\hline \multirow{3}{*}{$\begin{array}{l}\text { No-stress response } \\
\text { maintaining group }\end{array}$} & Frequency & 11 & 274 & \multirow{26}{*}{$18.58(7)^{* *}$} \\
\hline & $\begin{array}{l}\text { Expected } \\
\text { frequency }\end{array}$ & 25.85 & 259.15 & \\
\hline & Adj.residual & -3.67 & 3.67 & \\
\hline \multirow{3}{*}{$\begin{array}{l}\text { Low'stress response } \\
\text { maintaining group }\end{array}$} & Frequency & 19 & 145 & \\
\hline & $\begin{array}{l}\text { Expected } \\
\text { frequency }\end{array}$ & 14.88 & 149.12 & \\
\hline & Adj.residual & 1.23 & -1.23 & \\
\hline \multirow{3}{*}{$\begin{array}{l}\text { Low-stress response to } \\
\text { no- stress response } \\
\text { slow improvement } \\
\text { group }\end{array}$} & Frequency & 14 & 116 & \\
\hline & $\begin{array}{l}\text { Expected } \\
\text { frequency }\end{array}$ & 11.79 & 118.21 & \\
\hline & Adj.residual & 0.73 & -0.73 & \\
\hline \multirow{3}{*}{$\begin{array}{l}\text { Middle-stress response } \\
\text { to high-stress } \\
\text { response slow } \\
\text { deteriorated group }\end{array}$} & Frequency & 10 & 74 & \\
\hline & $\begin{array}{l}\text { Expected } \\
\text { frequency }\end{array}$ & 7.62 & 76.38 & \\
\hline & Adj.residual & 0.95 & -0.95 & \\
\hline \multirow{3}{*}{$\begin{array}{l}\text { Middle-stress response } \\
\text { to low-stress response } \\
\text { slow improvement } \\
\text { group }\end{array}$} & Frequency & 7 & 68 & \\
\hline & $\begin{array}{l}\text { Expected } \\
\text { frequency }\end{array}$ & 6.80 & 68.20 & \\
\hline & Adj.residual & 0.08 & -0.08 & \\
\hline \multirow{3}{*}{$\begin{array}{l}\text { Low-stress response to } \\
\text { no-stress response } \\
\text { rapid improvement } \\
\text { group }\end{array}$} & Frequency & 13 & 78 & \\
\hline & $\begin{array}{l}\text { Expected } \\
\text { frequency }\end{array}$ & 8.26 & 82.74 & \\
\hline & $A d j . r e s i d u a l$ & 1.82 & -1.82 & \\
\hline \multirow{3}{*}{$\begin{array}{l}\text { High-stress response } \\
\text { to low stress response } \\
\text { rapid improvement } \\
\text { group }\end{array}$} & Frequency & 4 & 62 & \\
\hline & $\begin{array}{l}\text { Expected } \\
\text { frequency }\end{array}$ & 5.99 & 60.01 & \\
\hline & $A d j . r e s i d u a l$ & -0.88 & 0.88 & \\
\hline \multirow{3}{*}{$\begin{array}{l}\text { Severe-stress response } \\
\text { maintaining group }\end{array}$} & Frequency & 7 & 35 & \\
\hline & $\begin{array}{l}\text { Expected } \\
\text { frequency }\end{array}$ & 3.81 & 38.19 & \\
\hline & Adj.residual & 1.75 & -1.75 & \\
\hline \multirow[b]{2}{*}{ Total } & Frequency & 85 & 852 & \\
\hline & $\begin{array}{l}\text { Expected } \\
\text { frequency }\end{array}$ & 85.0 & 852.0 & \\
\hline
\end{tabular}


following groups: the middle-stress response to high-stress response slow deteriorated group $(p<.05)$, high-stress response to low stress response rapid improvement group $(p<.01)$, and severe-stress response maintaining group $(p<.05)$. On the other hand, the subjects whose domiciles were significantly damaged are less likely to be in the no-stress response maintaining group $(p<.01)$, while the subjects who experienced their domiciles were half-collapsed or largely destroyed have the likelihood of belonging to the low-stress response to no- stress response slow improvement group $(p<.05)$ and the severe-stress response maintaining group $(p<.05)$. Furthermore, the subjects whose domiciles were partially destroyed are less likely to be in the severe-stress response maintaining group $(p<.05)$ while others whose domiciles were either slightly damaged or no damage did not indicate any statistical significance.

According to the result of residual analysis based on a cross-tabulation table between the cluster of experience of life outside a domicile and the cluster of chronological patterns, the subjects who had experienced living outside their domiciles have the most likelihood of

Table11. Abstract about Residual Analysis on Existence or Non-existence of The Dead / Missing Person in Family $(n=937)$ being in the low-stress response to no-stress response rapid improvement group $(p<.05)$ and the least likelihood of being in the no-stress response maintaining group $(p<.05)$. Meanwhile, the subjects who still lived outside their domiciles when the $1^{\text {st }}$ investigation took place are likely to be in the middle-stress response to high-stress response slow deteriorated group $(p<.01)$, and the severe-stress response maintaining group $(p<.05)$. On the other hand, those who never experienced living outside their domiciles are most likely to belong to the no-stress response maintaining group $(p<.01)$ and least likely to belong to the middle-stress response to high-stress response slow deteriorated group $(p<.05)$ or the high-stress response to low stress response rapid improvement group $(p<.05)$.

According to the residual analysis based on a cross-tabulation table between the cluster of having/not having missing or dead person(s) in the family and the cluster of chronological patterns, we found that the no-stress response maintaining group is mainly composed of the respondents who have few family members that are deceased or missing $(p<.01)$ or have nobody in the family who is deceased or missing $(p<.01)$.

\begin{tabular}{l|c|c}
\multicolumn{2}{c}{ Family $(n=937)$} \\
\hline \multirow{2}{*}{ There is } & Great frequency & Not available \\
\cline { 2 - 3 } & Low frequency & No-stress response maintaining group $* *$ \\
\hline \multirow{2}{*}{ There is not } & Great frequency & No-stress response maintaining group $* *$ \\
\cline { 2 - 3 } & Low frequency & Not available \\
\hline
\end{tabular}




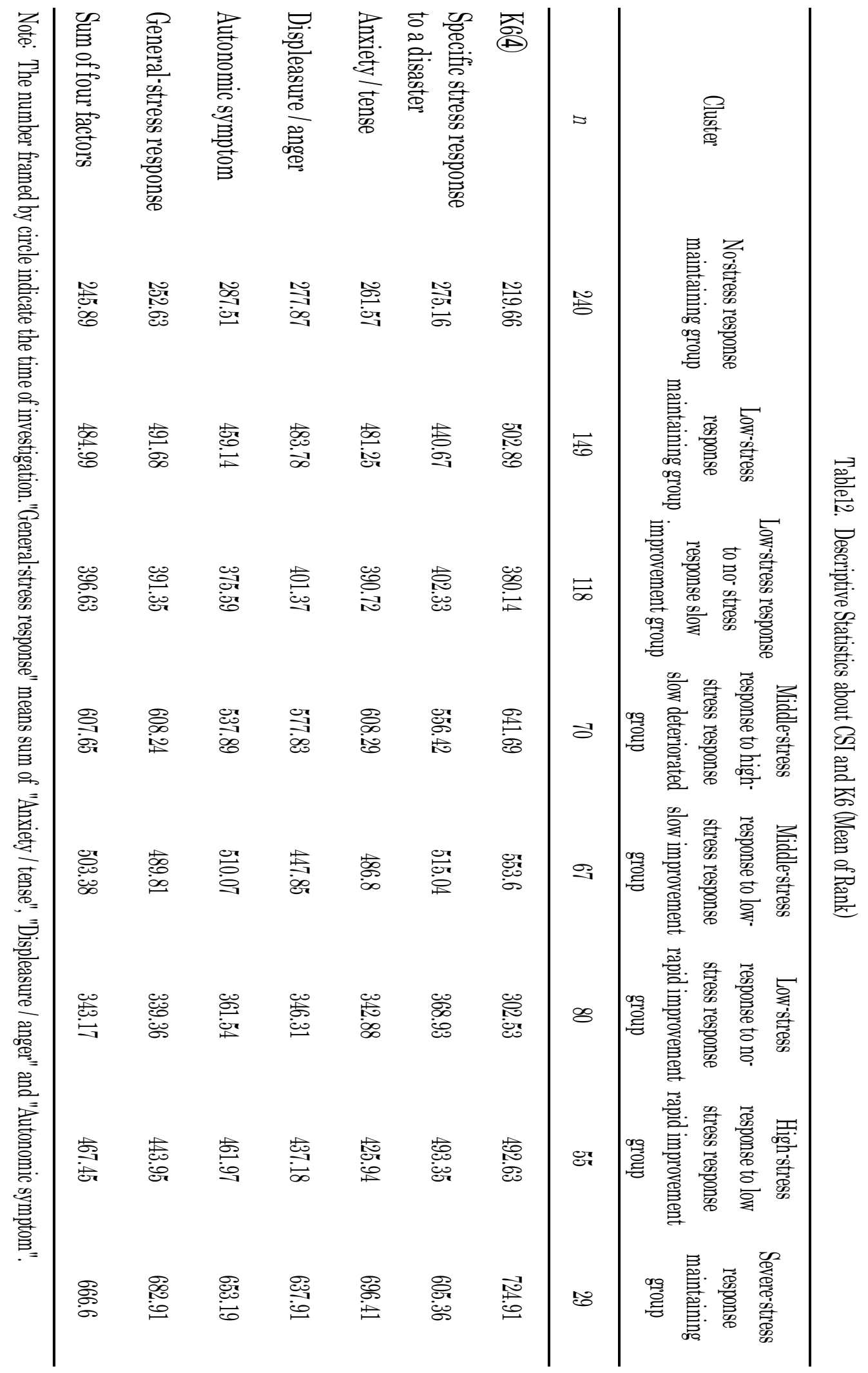


Lastly, a Kruskal-Wallis test was performed involving dependent variables - the scores of the K6 from the $4^{\text {th }}$ investigation, scores of the CSI (anxiety/tense, displeasure/anger, and autonomic symptoms, the sum of these three factors, specific stress response to the disaster, and the sum of all four factors). The purpose of this test was to identify how the chronological patterns of stress in the past are correlated with the current stress condition. The number of the subjects that were suitable for this test is 808 , and there were no missing values in all dependent variables.

The test result indicated that the effect of clusters was statistically significant in all dependent variables: $\mathrm{K} 6$ at the time of the $4^{\text {th }}$ investigation $\left(\chi^{2}=359.96, d f=7, p<.01\right)$, anxiety/tense $\left(\chi^{2}=223.42, d f=7, p<.01\right)$, displeasure/anger $\left(\chi^{2}=177.97, d f=7, p<.01\right)$, and autonomic symptoms $\left(\chi^{2}=156.87, d f=7, p\right.$ $<.01)$, the sum of the above three factors $\left(\chi^{2}=\right.$ $235.59, d f=7, p<.01$ ), specific stress response to the disaster $\left(\chi^{2}=159.50, d f=7, p<.01\right)$, and the sum of all four factors $\chi^{2}=240.68, d f=7$, $p<.01)$.

In this regard, we conducted multiple comparisons of those dependent variables in accordance with the Mann-Whitney U-test (the Type I error rate was 5\%). Table 12 shows the descriptive statistical results about stress among the clusters of CSI and K6. Next, the multiple comparison data are shown in Table 13 through

Table13. Result of Multiple Comparison about K6(4)

\begin{tabular}{|c|c|c|c|c|}
\hline Cluster & & $\begin{array}{c}\text { Number of } \\
\text { people }\end{array}$ & Mean of rank & Multiple comparison \\
\hline \multirow{8}{*}{ K6(4) } & $\begin{array}{l}\text { No-stress response } \\
\text { maintaining group (C1) }\end{array}$ & 240 & 219.66 & \multirow{8}{*}{$\mathrm{C} 1<\mathrm{C} 6, \mathrm{C} 3<\mathrm{C} 7, \mathrm{C} 2, \mathrm{C} 5, \mathrm{C} 4<\mathrm{C} 8$} \\
\hline & $\begin{array}{l}\text { Low-stress response } \\
\text { maintaining group (C2) }\end{array}$ & 149 & 502.89 & \\
\hline & $\begin{array}{l}\text { Low-stress response to no- } \\
\text { stress response slow } \\
\text { improvement group (C3) }\end{array}$ & 118 & 380.14 & \\
\hline & $\begin{array}{l}\text { Middle-stress response to } \\
\text { high-stress response slow } \\
\text { deteriorated group (C4) }\end{array}$ & 70 & 641.69 & \\
\hline & $\begin{array}{l}\text { Middle-stress response to } \\
\text { low-stress response slow } \\
\text { improvement group (C5) }\end{array}$ & 67 & 553.6 & \\
\hline & $\begin{array}{l}\text { Low-stress response to no- } \\
\text { stress response rapid } \\
\text { improvement group }(\mathrm{C} 6)\end{array}$ & 80 & 302.53 & \\
\hline & $\begin{array}{l}\text { High-stress response to } \\
\text { low stress response rapid } \\
\text { improvement group }(\mathrm{C} 7)\end{array}$ & 55 & 492.63 & \\
\hline & $\begin{array}{l}\text { Severe-stress response } \\
\text { maintaining group }(\mathrm{C} 8)\end{array}$ & 29 & 724.91 & \\
\hline Total & & 808 & & \\
\hline
\end{tabular}

Note: The number framed by circle indicate the time of investigation. 
Table 19. Each cluster of stress response will be expediently described in abbreviation as follows: $\mathrm{C} 1$ as no-stress response maintaining group, C2 as low-stress response maintaining group, C3 as low-stress response to no- stress response slow improvement group, $\mathrm{C} 4$ as middle-stress response to high-stress response slow deteriorated group, C5 as middle-stress response to low-stress response slow improvement group, C6 as low-stress response to no-stress response rapid improvement group, C7 as high-stress response to low stress response rapid improvement group, and C8 as severe-stress response maintaining group.

According to multiple comparisons involving
K6 scores at the $4^{\text {th }}$ investigation, the no-stress response maintaining group (C1) significantly has the lowest mean value among all the stress response clusters. Also, the low-stress response to no-stress response rapid improvement group (C6) and the low-stress response to no- stress response slow improvement group (C3) had significantly lower mean values than the high-stress response to low stress response rapid improvement group (C7), the low-stress response maintaining group (C2), the middle-stress response to low-stress response slow improvement group (C5), the middle-stress response to high-stress response slow deteriorated group (C4), and the

Table14. Result of Multiple Comparison about Specific Stress Response to a Disaster

\begin{tabular}{|c|c|c|c|c|}
\hline Cluster & & $\begin{array}{c}\text { Number of } \\
\text { people }\end{array}$ & Mean of rank & Multiple comparison \\
\hline \multirow{8}{*}{$\begin{array}{c}\text { Specific } \\
\text { stress } \\
\text { response to a } \\
\text { disaster }\end{array}$} & $\begin{array}{l}\text { No-stress response } \\
\text { maintaining group }(\mathrm{C} 1)\end{array}$ & 240 & 275.16 & \multirow{8}{*}{$\mathrm{C} 1<\mathrm{C} 6, \mathrm{C} 3, \mathrm{C} 2, \mathrm{C} 7, \mathrm{C} 5<\mathrm{C} 4, \mathrm{C} 8$} \\
\hline & $\begin{array}{l}\text { Low-stress response } \\
\text { maintaining group }(\mathrm{C} 2)\end{array}$ & 149 & 440.67 & \\
\hline & $\begin{array}{l}\text { Low־stress response to no- } \\
\text { stress response slow } \\
\text { improvement group (C3) }\end{array}$ & 118 & 402.33 & \\
\hline & $\begin{array}{l}\text { Middle-stress response to } \\
\text { high-stress response slow } \\
\text { deteriorated group (C4) }\end{array}$ & 70 & 556.42 & \\
\hline & $\begin{array}{l}\text { Middle-stress response to } \\
\text { low-stress response slow } \\
\text { improvement group (C5) }\end{array}$ & 67 & 515.04 & \\
\hline & $\begin{array}{l}\text { Low-stress response to no- } \\
\text { stress response rapid } \\
\text { improvement group }(\mathrm{C} 6)\end{array}$ & 80 & 368.93 & \\
\hline & $\begin{array}{l}\text { High-stress response to } \\
\text { low stress response rapid } \\
\text { improvement group }(\mathrm{C} 7)\end{array}$ & 55 & 493.35 & \\
\hline & $\begin{array}{l}\text { Severe-stress response } \\
\text { maintaining group }(\mathrm{C} 8)\end{array}$ & 29 & 605.36 & \\
\hline Total & & 808 & & \\
\hline
\end{tabular}


severe-stress response maintaining group (C8). Furthermore, the mean values of the high-stress response to low stress response rapid improvement group (C7), the low-stress response maintaining group (C2), the middle-stress response to low-stress response slow improvement group (C5), and the middle-stress response to high-stress response slow deteriorated group (C4) are significantly lower than that of the severe-stress response maintaining group (C8). In particular, C7 and $\mathrm{C} 2$ have significantly lower mean values than that of $\mathrm{C} 4$.

According to multiple comparisons involving specific stress response to a disaster in CSI, the no-stress response maintaining group (C1) significantly has the lowest mean value across all stress response clusters. Also, the low-stress response to no-stress response rapid improvement group (C6), the low-stress response to no- stress response slow improvement group (C3), the low-stress response maintaining group (C2), the high-stress response to low stress response rapid improvement group (C7), and the middle-stress response to low-stress response slow improvement group (C5) have significantly lower mean values than the middle-stress response to high-stress response slow deteriorated group (C4) and the severe-stress response maintaining group (C8). In particular, C6 has a significantly low mean

Table15. Result of Multiple Comparison about Anxiety / tense

\begin{tabular}{|c|c|c|c|c|}
\hline Cluster & & $\begin{array}{c}\text { Number of } \\
\text { people }\end{array}$ & Mean of rank & Multiple comparison \\
\hline \multirow{8}{*}{$\begin{array}{l}\text { Anxiety / } \\
\text { tense }\end{array}$} & $\begin{array}{l}\text { No-stress response } \\
\text { maintaining group }(\mathrm{C} 1)\end{array}$ & 240 & 261.57 & \multirow{8}{*}{$\begin{array}{cl}\text { Note: } & \mathrm{C} 1<\mathrm{C} 3, \mathrm{C} 7, \mathrm{C} 2, \mathrm{C} 5 \\
& \mathrm{C} 6, \mathrm{C} 3<\mathrm{C} 2, \mathrm{C} 5\end{array}$} \\
\hline & $\begin{array}{l}\text { Low-stress response } \\
\text { maintaining group }(\mathrm{C} 2)\end{array}$ & 149 & 481.25 & \\
\hline & $\begin{array}{l}\text { Low-stress response to no- } \\
\text { stress response slow } \\
\text { improvement group (C3) }\end{array}$ & 118 & 390.72 & \\
\hline & $\begin{array}{l}\text { Middle-stress response to } \\
\text { high-stress response slow } \\
\text { deteriorated group }(\mathrm{C} 4)\end{array}$ & 70 & 608.29 & \\
\hline & $\begin{array}{l}\text { Middle-stress response to } \\
\text { low-stress response slow } \\
\text { improvement group (C5) }\end{array}$ & 67 & 486.8 & \\
\hline & $\begin{array}{l}\text { Low-stress response to no- } \\
\text { stress response rapid } \\
\text { improvement group (C6) }\end{array}$ & 80 & 342.88 & \\
\hline & $\begin{array}{l}\text { High-stress response to } \\
\text { low stress response rapid } \\
\text { improvement group (C7) }\end{array}$ & 55 & 425.94 & \\
\hline & $\begin{array}{l}\text { Severe-stress response } \\
\text { maintaining group }(\mathrm{C} 8)\end{array}$ & 29 & 696.41 & \\
\hline Total & & 808 & & \\
\hline
\end{tabular}


value in comparison to $\mathrm{C} 7$ and $\mathrm{C5}$.

From the multiple comparisons involving anxiety/tense in CSI, the mean values of the no-stress response maintaining group $(\mathrm{C} 1)$, the low-stress response to no-stress response rapid improvement group (C6), the low-stress response to no- stress response slow improvement group (C3), the high-stress response to low stress response rapid improvement group (C7), the low-stress response maintaining group $(\mathrm{C} 2)$, and the middle-stress response to low-stress response slow improvement group (C5) are significantly low in comparison to the mean values of the middle-stress response to high-stress response slow deteriorated group (C4) and the severe-stress response maintaining group (C8). In particular, $\mathrm{C} 1$ indicates the significantly lower mean value than $\mathrm{C} 3, \mathrm{C} 7, \mathrm{C} 2$, and $\mathrm{C} 5$. Furthermore, the mean values of C6 and C3 are also significantly lower than C2 and C5.

In terms of multiple comparisons involving displeasure/anger in CSI, the mean values of the no-stress response maintaining group (C1), the low-stress response to no-stress response rapid improvement group (C6), the low-stress response to no- stress response slow improvement group (C3), the high-stress response to low stress response rapid improvement group (C7), the middle-stress

Table16. Result of Multiple Comparison about Displeasure / anger

\begin{tabular}{|c|c|c|c|c|}
\hline Cluster & & $\begin{array}{c}\text { Number of } \\
\text { people }\end{array}$ & Mean of rank & Multiple comparison \\
\hline \multirow{8}{*}{$\begin{array}{l}\text { Displeasure / } \\
\text { anger }\end{array}$} & $\begin{array}{l}\text { No-stress response } \\
\text { maintaining group (C1) }\end{array}$ & 240 & 277.87 & \multirow{8}{*}{$\begin{array}{c}\mathrm{C} 1, \mathrm{C} 6, \mathrm{C} 3, \mathrm{C} 7, \mathrm{C} 5, \mathrm{C} 2<\mathrm{C} 4, \mathrm{C} 8 \\
\\
\text { Note: } \\
\mathrm{C} 1<\mathrm{C} 3, \mathrm{C} 7, \mathrm{C} 5, \mathrm{C} 2 \\
\mathrm{C} 6, \mathrm{C} 3<\mathrm{C} 2\end{array}$} \\
\hline & $\begin{array}{l}\text { Low-stress response } \\
\text { maintaining group (C2) }\end{array}$ & 149 & 483.78 & \\
\hline & $\begin{array}{l}\text { Low-stress response to no- } \\
\text { stress response slow } \\
\text { improvement group (C3) }\end{array}$ & 118 & 401.37 & \\
\hline & $\begin{array}{l}\text { Middle-stress response to } \\
\text { high-stress response slow } \\
\text { deteriorated group (C4) }\end{array}$ & 70 & 577.83 & \\
\hline & $\begin{array}{l}\text { Middle-stress response to } \\
\text { low-stress response slow } \\
\text { improvement group (C5) }\end{array}$ & 67 & 447.85 & \\
\hline & $\begin{array}{l}\text { Low-stress response to no- } \\
\text { stress response rapid } \\
\text { improvement group }(\mathrm{C} 6)\end{array}$ & 80 & 346.31 & \\
\hline & $\begin{array}{l}\text { High-stress response to } \\
\text { low stress response rapid } \\
\text { improvement group (C7) }\end{array}$ & 55 & 437.18 & \\
\hline & $\begin{array}{l}\text { Severe-stress response } \\
\text { maintaining group (C8) }\end{array}$ & 29 & 637.91 & \\
\hline Total & & 808 & & \\
\hline
\end{tabular}


response to low-stress response slow improvement group (C5), and the low-stress response maintaining group (C2) are significantly low in comparison to the mean values of the middle-stress response to high-stress response slow deteriorated group (C4) and the severe-stress response maintaining group (C8). In particular, C1 indicates a significantly lower mean value than $\mathrm{C} 3, \mathrm{C} 7, \mathrm{C} 5$, and $\mathrm{C} 2$. Furthermore, the mean values of C6 and C3 are also significantly lower than the mean values of $\mathrm{C} 2$.

From the multiple comparisons regarding autonomic symptoms in CSI, the mean values of the no-stress response maintaining group
(C1), the low-stress response to no-stress response rapid improvement group (C6), and the low-stress response to no- stress response slow improvement group (C3) are significantly low in comparison to the mean values of the low-stress response maintaining group (C2), the high-stress response to low stress response rapid improvement group (C7), the middle-stress response to low-stress response slow improvement group (C5), the middle-stress response to high-stress response slow deteriorated group (C4), and the severe-stress response maintaining group (C8). In particular, the mean value of $\mathrm{C} 1$ is significantly lower than that of C3. Similarly,

Tbale17. Result of Multiple Comparison about Autonomic symptom

\begin{tabular}{|c|c|c|c|c|}
\hline Cluster & & $\begin{array}{c}\text { Number of } \\
\text { people }\end{array}$ & Mean of rank & Multiple comparison \\
\hline \multirow{8}{*}{$\begin{array}{l}\text { Autonomic } \\
\text { symptom }\end{array}$} & $\begin{array}{l}\text { No-stress response } \\
\text { maintaining group }(\mathrm{C} 1)\end{array}$ & 240 & 287.51 & \multirow{8}{*}{$\begin{array}{ll}\text { Note: } & \mathrm{C} 1<\mathrm{C} 3 \\
& \mathrm{C} 2, \mathrm{C} 7, \mathrm{C} 5<\mathrm{C} 8\end{array}$} \\
\hline & $\begin{array}{l}\text { Low-stress response } \\
\text { maintaining group (C2) }\end{array}$ & 149 & 459.14 & \\
\hline & $\begin{array}{l}\text { Low-stress response to no- } \\
\text { stress response slow } \\
\text { improvement group }(\mathrm{C} 3)\end{array}$ & 118 & 375.59 & \\
\hline & $\begin{array}{l}\text { Middle-stress response to } \\
\text { high-stress response slow } \\
\text { deteriorated group }(\mathrm{C} 4)\end{array}$ & 70 & 537.89 & \\
\hline & $\begin{array}{l}\text { Middle-stress response to } \\
\text { low-stress response slow } \\
\text { improvement group }(\mathrm{C} 5)\end{array}$ & 67 & 510.07 & \\
\hline & $\begin{array}{l}\text { Low-stress response to no- } \\
\text { stress response rapid } \\
\text { improvement group }(\mathrm{C} 6)\end{array}$ & 80 & 361.54 & \\
\hline & $\begin{array}{l}\text { High-stress response to } \\
\text { low stress response rapid } \\
\text { improvement group }(\mathrm{C} 7)\end{array}$ & 55 & 461.97 & \\
\hline & $\begin{array}{l}\text { Severe-stress response } \\
\text { maintaining group }(\mathrm{C} 8)\end{array}$ & 29 & 653.19 & \\
\hline Total & & 808 & & \\
\hline
\end{tabular}


$\mathrm{C} 2, \mathrm{C} 7$, and $\mathrm{C} 5$ each have a significantly lower value than $\mathrm{C} 8$.

From the multiple comparisons in the sum of three stress response factors regarding the general-stress response of the CSI, the no-stress response maintaining group $(\mathrm{C} 1)$ indicates the lowest mean value across all clusters. Also, the low-stress response to no-stress response rapid improvement group (C6), the low-stress response to no- stress response slow improvement group (C3), the high-stress response to low stress response rapid improvement group (C7), the middle-stress response to low-stress response slow improvement group (C5), and the low-stress response maintaining group (C2) have significantly lower mean values than the middle-stress response to high-stress response slow deteriorated group (C4) and the severe-stress response maintaining group (C8). In particular, the mean values of $\mathrm{C} 6$ and $\mathrm{C} 3$ are significantly lower than those of $\mathrm{C} 5$, and $\mathrm{C} 2$.

From the multiple comparisons in the sum of all four stress response factors in CSI, the no-stress response maintaining group (C1) indicates the lowest mean value across all clusters. In particular, the low-stress response to no-stress response rapid improvement group (C6) has a significantly lower mean value than those of the high-stress response to low stress

Table18. Result of Multiple Comparison about General-stress response

\begin{tabular}{|c|c|c|c|c|}
\hline Cluster & & $\begin{array}{c}\text { Number of } \\
\text { people }\end{array}$ & Mean of rank & Multiple comparison \\
\hline \multirow{8}{*}{$\begin{array}{l}\text { General- } \\
\text { stress } \\
\text { response }\end{array}$} & $\begin{array}{l}\text { No-stress response } \\
\text { maintaining group (C1) }\end{array}$ & 240 & 252.63 & \multirow{8}{*}{$\mathrm{C} 1<\mathrm{C} 6, \mathrm{C} 3, \mathrm{C} 7, \mathrm{C} 5, \mathrm{C} 2<\mathrm{C} 4, \mathrm{C} 8$} \\
\hline & $\begin{array}{l}\text { Low-stress response } \\
\text { maintaining group }(\mathrm{C} 2)\end{array}$ & 149 & 491.68 & \\
\hline & $\begin{array}{l}\text { Low-stress response to no- } \\
\text { stress response slow } \\
\text { improvement group (C3) }\end{array}$ & 118 & 391.35 & \\
\hline & $\begin{array}{l}\text { Middle-stress response to } \\
\text { high-stress response slow } \\
\text { deteriorated group (C4) }\end{array}$ & 70 & 608.24 & \\
\hline & $\begin{array}{l}\text { Middle-stress response to } \\
\text { low-stress response slow } \\
\text { improvement group (C5) }\end{array}$ & 67 & 489.81 & \\
\hline & $\begin{array}{l}\text { Low-stress response to no- } \\
\text { stress response rapid } \\
\text { improvement group (C6) }\end{array}$ & 80 & 339.36 & \\
\hline & $\begin{array}{l}\text { High-stress response to } \\
\text { low stress response rapid } \\
\text { improvement group (C7) }\end{array}$ & 55 & 443.95 & \\
\hline & $\begin{array}{l}\text { Severe-stress response } \\
\text { maintaining group (C8) }\end{array}$ & 29 & 682.91 & \\
\hline Total & & 808 & & \\
\hline
\end{tabular}


response rapid improvement group (C7), the low-stress response maintaining group $(\mathrm{C} 2)$, the middle-stress response to low-stress response slow improvement group (C5), the middle-stress response to high-stress response slow deteriorated group (C4), and the severe-stress response maintaining group (C8). The mean value of the low-stress response to no- stress response slow improvement group (C3) is significantly lower than the mean values of C2, C5, C4, and C8. Similarly, the mean value of the high-stress response to low stress response rapid improvement group (C7) is significantly lower than the mean values $\mathrm{C} 4$ and $\mathrm{C} 8$.

\section{Discussion}

The main purpose of this study is to focus on local government workers who perform the central roles for recovery and restoration work after the Great East Japan Earthquake and to investigate the different types of stress that they reported in chronological order. In particular, we classified each stress type into chronological patterns based on survey answers provided by the public servants and examined how the initial condition of their disaster experiences and their attributes are related to the subsequent patterns of stress. Furthermore, the correlation between the participants' current

Table19. Result of Multiple Comparison about Sum of four factors

\begin{tabular}{|c|c|c|c|c|}
\hline Cluster & & $\begin{array}{c}\text { Number of } \\
\text { people }\end{array}$ & Mean of rank & Multiple comparison \\
\hline \multirow{8}{*}{$\begin{array}{l}\text { Sum of four } \\
\text { factors }\end{array}$} & $\begin{array}{l}\text { No-stress response } \\
\text { maintaining group (C1) }\end{array}$ & 240 & 245.89 & \multirow{9}{*}{ 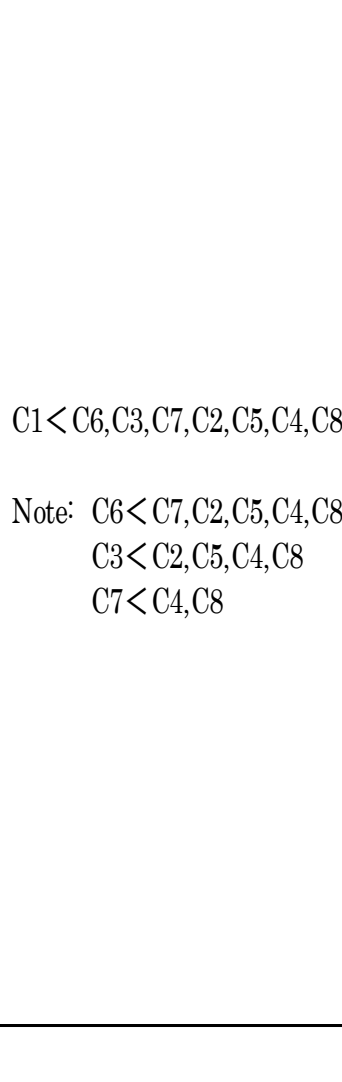 } \\
\hline & $\begin{array}{l}\text { Low-stress response } \\
\text { maintaining group (C2) }\end{array}$ & 149 & 484.99 & \\
\hline & $\begin{array}{l}\text { Low-stress response to no- } \\
\text { stress response slow } \\
\text { improvement group (C3) }\end{array}$ & 118 & 396.63 & \\
\hline & $\begin{array}{l}\text { Middle-stress response to } \\
\text { high-stress response slow } \\
\text { deteriorated group (C4) }\end{array}$ & 70 & 607.65 & \\
\hline & $\begin{array}{l}\text { Middle-stress response to } \\
\text { low-stress response slow } \\
\text { improvement group (C5) }\end{array}$ & 67 & 503.38 & \\
\hline & $\begin{array}{l}\text { Low-stress response to no- } \\
\text { stress response rapid } \\
\text { improvement group (C6) }\end{array}$ & 80 & 343.17 & \\
\hline & $\begin{array}{l}\text { High-stress response to } \\
\text { low stress response rapid } \\
\text { improvement group (C7) }\end{array}$ & 55 & 467.45 & \\
\hline & $\begin{array}{l}\text { Severe-stress response } \\
\text { maintaining group (C8) }\end{array}$ & 29 & 666.6 & \\
\hline Total & & 808 & & \\
\hline
\end{tabular}


observed stress at each point and the chronological pattern of stress is also thoroughly investigated.

The first objective of this study, the classification of chronological patterns of stress, will be discussed. We initially explain the reason for performing a cluster analysis of $\mathrm{K} 6$ along with two additional variables. $\mathrm{K} 6$ is an instrument of stress response scales, consisting of 6 items, and it was originally developed in consideration of the psychological impacts on the public servants who have been struck by natural disaster. However, the efficiency of K6 was questioned due to concern that only limited types of stress responses could possibly be measured, and because of this concern, an extra two variables- Asleep condition and communication with co-workers-were added to the original $\mathrm{K} 6$.

Our test results show that scores on the items examining sleep and communication with co-workers and the scores of the original K6 were almost comparable. Especially, asleep condition was identified as an important variable for measurement of one's stress level. Chronological patterns of stress involving those variables are classified into 8 clusters. The rate of each cluster is as follows: $30.57 \%$ no-stress response maintaining group; $17.65 \%$ low-stress response maintaining group; $13.66 \%$ low-stress response to no- stress response slow improvement group; $9.14 \%$ middle-stress response to high-stress response slow deteriorated group; $7.98 \%$ middle-stress response to low-stress response slow improvement group; $9.66 \%$ low-stress response to no-stress response rapid improvement group; 6.93\% high-stress response to low stress response rapid improvement group; and $4.41 \%$ severe-stress response maintaining group. In particular, two groups - the middle-stress response to high-stress response slow deteriorated group $(9.14 \%)$ and the severe-stress response maintaining group $(4.41 \%)$ - indicated the highest stress values among stress group clusters at the $3^{\text {rd }}$ investigation. This implies that $13.55 \%$ of local government workers remained at a high level of stress 15 months after the earthquake. From these findings, it also indicates that $67.44 \%$ of the respondents who were in high stress felt that their stress was even elevating chronologically. In order to explain this phenomenon, implications from the second purpose of our study can be discussed now.

From our second objective, an investigation about how the initial condition of the public servants' disaster experiences and their attributes are related to the subsequent patterns of stress, the middle-stress response to high-stress response slow deteriorated group and the severe-stress response maintaining group are correlated with domicile damage and living outside their domiciles. In addition to the two clusters as mentioned above, the cluster that contains the highest proportion of people whose domiciles were completely destroyed is the high-stress response to low-stress response rapid improvement group. However, those two groups are different from the high-stress response to low-stress response rapid improvement group from the viewpoint that 
people in the former groups have a high tendency of prolonged living outside their domiciles at the time of the first investigation. From this finding, one's experience of living outside one's domicile is considered as an important variable in order to determine the likelihood of one's continued improvement in the face of stress that is induced by the disaster. Also, members of the no-stress response maintaining group are least likely to have family member(s) who are dead or missing.

Meanwhile, no significance of stress response in correlation with disaster-related duties and overtime work was found. However, these items were asked only once at the $1^{\text {st }}$ investigation. As for questions involving overtime, they asked participants whether they worked more or fewer than 100 hours of work, and the lack of identified significance is possibly due to some methodological errors. For the future direction of this continuing research, it is necessary to add more items that allow participants to describe recollections of their experiences in the survey form.

Lastly, the correlation between the current stress of the local government workers and the chronological patterns of stress, which is our $3^{\text {rd }}$ investigation objective, will be discussed. In connection to $\mathrm{K} 6$ at the time of the $4^{\text {th }}$ investigation, clusters including the severe-stress response maintaining group and the middle-stress response to high-stress response slow deteriorated group indicated the highest stress values, which are composed of $12.25 \%$ of survey participants.

With regard to $\mathrm{K} 6$ at the time of the $4^{\text {th }}$ investigation, score of severe-stress response maintaining group (C8) and middle-stress response to high-stress response slow deteriorated group (C4) are worst. And middle-stress response to low-stress response slow improvement group (C5), low-stress response maintaining group (C2), high-stress response to low-stress response rapid improvement group (C7), low-stress response to no-stress response slow improvement group (C3), low-stress response to no-stress response rapid improvement group (C6), and no-stress response maintaining group (C1) are follow them. Specifically C3, C6, and $\mathrm{C} 1$ indicate low score in any variables, thus they are clusters which have low stress response.

As for each factors of CSI, score of severe-stress response maintaining group (C8) and middle-stress response to high-stress response slow deteriorated group (C4) are worst. And middle-stress response to low-stress response slow improvement group (C5) and high-stress response to low-stress response rapid improvement group (C7) are relatively high score in specific stress response to a disaster. $\mathrm{C} 5$ and $\mathrm{C} 7$ had high stress response at the outset of disaster. The fact suggests that specific stress response to a disaster relate with state at the outset of disaster.

In terms of anxiety / tense, score of severe-stress response maintaining group (C8) and middle-stress response to high-stress response slow deteriorated group (C4) are worst. And middle-stress response to low-stress response slow improvement group (C5) and low-stress response maintaining group $(\mathrm{C} 2)$ are 
relatively high score in anxiety / tense. This order of score corresponds to patterns in $\mathrm{K} 6$ score at $3^{\text {rd }}$ investigation and $4^{\text {th }}$ investigation. Therefore, anxiety / tense have stronger relationship with recent conditions.

In terms of displeasure / anger, score of severe-stress response maintaining group (C8) and middle-stress response to high-stress response slow deteriorated group (C4) are worst. Low-stress response maintaining group (C2) is follow. As previously noted, C2 indicates relatively minor stress response at the outset of disaster. In view of this, displeasure / anger have relationship stronger relationship with recent conditions too.

In terms of autonomic symptom, severe-stress response maintaining group (C8) is worst. Middle-stress response to high-stress response slow deteriorated group (C4), middle-stress response to low-stress response slow improvement group (C5), high-stress response to low-stress response rapid improvement group (C7), and Low-stress response maintaining group $(\mathrm{C} 2)$ are follow. $\mathrm{C} 7$ which had high stress response at $1^{\text {st }}$ investigation have relatively high score of autonomic symptom score and specific stress response to a disaster. This common point means that autonomic symptom, along with specific stress response to a disaster, relate with state at the outset of disaster.

In terms of general-stress response (sum of "anxiety / tense", "displeasure / anger", and "autonomic symptom) and PTSD-like response (sum of four factors), severe-stress response maintaining group (C8) and middle-stress response to high-stress response slow deteriorated group (C4) are worst, and low-stress response maintaining group (C2) and middle-stress response to low-stress response slow improvement group (C5) indicate relatively high score. The order of score is common in both clusters. This common point means that the score of general-stress response is able to drive up the score of PTSD-like response. Result in this study suggests that the upthrust might make expertise "PTSD develops late" too much.

\section{General overview}

First, one of the risk factor in disaster which is able to threat mental health relates circumstances in the aftermath of the disaster (e.g. damage of domicile / experience of life outside domicile). On the other hand, despite the overall mean score of $\mathrm{K} 6$ decreased with time, there is the cluster whose stress response increased about $+1 \mathrm{SD}$ from $2^{\text {nd }}$ investigation to $3^{\text {rd }}$ investigation. In foregoing cluster, worsening of stress response might be caused by recent conditions. These results indicate that support persons need to look around both circumstances in the aftermath of the disaster and current conditions.

Second, instruments which are used for assess mental health of disaster victims such as IES-R (Weiss \& Marmer, 1997) include items that measure a similar general stress response. To avoid confusion with general-stress response in assessment of PTSD-like response, scales for PTSD-like response would preferably be focused on factors that are related to one's 
experience and memory. If an individual demonstrates a high stress level in the middle of the disaster recovery process, detailed examination of his or her current conditions of stress would be clinically effective.

Local government workers are required to conduct restorative work starting early in the aftermath of the disaster and to continue these activities for a medium-term to long-term span. Problem of local government workers who have been impacted by a disaster which were recognized in Wakashima, Kozukua, \& Noguchi (2014), such as heavy burdens of restorative work which increases with time, disparity of workload, uncertainty for the future, and rift with co-worker and family members, can become stressor. In other words, to care for local government workers, support persons have to consider not only direct impact of disaster but also problems caused by disaster indirectly.

\section{Acknowledgments}

This works was supported by JSPS KAKENHI Grant-in-Aid for Scientific Research (C) Number 25380915

\section{References}

Asai, K., Morikawa, N., Hiraizumi, T., Usami, T., \& Wakashima, K. 2013 Development of comprehensive stress response inventory. Annual Report, Graduate School of Education, Tohoku University, 62(1), 283-302. [ in Japanese ] (浅井継悟 - 森川夏乃 - 平泉 拓 - 宇佐美貴章 - 若島孔文 (2013) 包括 的ストレス尺度作成の試み 東北大学大
学院教育学研究科研究年報, 62(1), 283-302.

Furukawa, T., Ono, Y., Uda, H., \& Nakane, Y. 2003 Ippan jinkou chuno kanbenna sukurininguni kannsuru kennkyu (Study of Convenient Assessment of Mental Disease in General Population). Research report of Health and Labour Sciences Research Grant in 2002. [ in Japanese ] (古川壽亮 - 大野裕 • 宇田英典・中根允文 (2003) 一般人口中 の精神疾患の簡便なスクリーニングに関 寸る研究平成 14 年度厚生労働科学研 究費補助金 (厚生労働科学特別研究事業) 心の健康問題と対策基盤の実態に関する 研究 研究協力報告書 )

Kessler, R.C., Andrews, G., Colpe, L.J., Hiripi, E., Mroczek, D.K., Normand, S.L., Walters, E.E., \& Zaslavsky, A.M. 2002 Short screening scales to monitor population prevalences and trends in non-specific psychological distress. Psychological Medicine 32 (6), 959-976.

Wakashima,K., Kozuka,T., \& Noguchi,S. 2014 Jichitaisyokuin no mental support (Mental Support for Local Government Workers) Governance, 155, 28-30. [ in Japanese ] (若島孔文・狐塚貴博 - 野口修司 (2014) 自治体職員のメンタルサポート

Governance, 155, 28-30. ぎょうせい

Wakashima,K., Kozuka,T., Noguchi,S., Kobayashi, T., \& Hasegawa, K. 2012 Higashi Nihon Daishinsai ni kakawarru ishinomakishi syokuin ni kansuru kenkouchosa houkoku (Report about Health Survey of Ishinomaki City Government Workers (1) ). Higashi Nihon Daishinsai PTG 
Mental Support Institute. [ in Japanses ] (若 島孔文・狐塚貴博 - 野口修司 - 小林 智 長谷川啓三 (2012) 東日本大震災に関わ る石巻市役所職員に関する健康調查報告 (1) 東日本大震災 PTG 心理支援機構

Wakashima, K. \& Noguchi, S. 2013 Gyousei syokuineno shinrisyakaishien(1) - Ishinomaki shiyakusyo syokuineno approach. Hasegawa, K. \& Wakashima, K. Eds. 2013 A guidebook for psychosocial support in earthquake disaster. Tokyo: Kanekoshobo. [ in Japanese ] （若島孔文・野口修司 (2013) 行政職員の 心理社会支援(1) - 石巻市役所職員へのア プローチ 長谷川啓三・若島孔文(編) 震災 心理社会支援ガイドブック - 東日本大震 災における現地基幹大学を中心にした実 践から学ぶ- 金子書房

Weiss, D. S., \& Marmar, C. R., (1997). The Impact of Event Scale-Revised. In Wilson,J.P., \& Keane, T. M. (Eds.), Assessing psychological trauma and PTSD: A practitioner's handbook (pp. 399-411). New York: Guilford Press. 